\title{
Chronic Respiratory Diseases, Lung Microbiome and Imaging Novelties
}

R. Rodriguez-Roisin, MD, PhD, FRCP, FERS

In the previous issue 2017 of BRN Reviews, one good example of the complex challenge imposed to the global respiratory community by the impact of chronic obstructive pulmonary disease (COPD) in Latin America was exemplified by the findings of the PLATINO Study (Montes de Oca M and Lopez-Varela MV, BRN Rev 2017;3:3-17), akin to the epidemiological concepts of the very recent release of the Global Initiative of Chronic Obstructive Pulmonary Disease (GOLD) 2017 Report ${ }^{1}$. In the current issue, we further illustrate the information on the globalization of COPD through the unique description of several of the differential features that characterize COPD in China. The authors, Wen Fuqiang, a leading Chinese clinical respiratory expert, and Yongchun Shen draw our attention to the fact that China has the largest population of elderly and smokers in the world, so remaining the country with the biggest number of COPD patients so far. Multiple risk factors, such as massive smoking, and enormous amounts of inhaled biomass fuel and air pollution greatly contribute to this terrible situation which leads to a vast mortality. Yet, some promising advances in the prevention and treatment of COPD have already begun, although both early diagnosis and use of spirometry should be more intensely widespread. Importantly, new strategies and creative actions to improve disease management are being actively implemented.

The second chronic respiratory disorder herein addressed is idiopathic pulmonary fibrosis (IPF), a chronic progressive interstitial disease of the lungs, whose incidence has increased over time in most countries around the world with a current range of 2.8-9.3 per 100,000 persons per year. Multiple medications have been unsuccessfully explored for the treatment of IPF so far. However, only pirfenidone and nintedanib appear to be currently recommended by international experts to slow the progression of the disease. The review written by Xiaoping Wu, Robert Kaner, and Fernando J. Martinez, a well-known worldwide expert in the field, points to the medications that have been evaluated as treatments for IPF over the last decades, including the currently 
recommended alluded to agents. A complementary view on ongoing clinical trials and future directions is also considered.

The emerging term microbiome describes either the collective genomes of the microorganisms that reside in an environmental niche or the microorganisms themselves. Also named microbiota, this lung model encompasses the collection of microorganisms that live in and on the human body, and the advent of newer technologies facilitates its investigation. Manoj Mammen and Sanjay Sethi, a world expert in lung infections related to chronic airways disease, provide a nice overview of the recent knowledge on the lung microbiome in common chronic airways diseases, such as COPD, bronchiectasis and bronchial asthma in both stable and acute conditions. And the introduction of newer advantageous technologies opens new ways of research of this area. A better understanding of the impact of these new concepts using new robust technologies on the pathogenesis, progression and aggravation of chronic lung disease may most likely lead to future microbiome-related therapies. Indeed, in the previous article on IPF, a mention is already made to the potential therapeutic advantages derived from these new microbiota concepts.

The fourth article is linked to recent promising innovative radiographic findings in the field of medical imaging of chronic airway and interstitial lung parenchyma diseases. This review highlights the utilization of computational power to facilitate the quantitative analysis of imaging, especially computed tomography, to further enhance the vision of several chronic respiratory diseases. This enthralling review that includes a series of remarkable illustrations is written by Samuel Ash and George Washko, the latter known to have a renowned international solid experience in this field. These new imaging technologies may help to facilitate not only our understanding of the mechanisms underlying chronic lung diseases, but also their potential therapies.

Last but not least, pulmonary hypertension, which is another life-threatening chronic pulmonary vascular disease and referred to as a mean pulmonary artery pressure $\geq 25 \mathrm{mmHg}$, is a common complication of cardiac and respiratory diseases and primarily affects the heart by increasing right ventricular afterload. The initial response of the right ventricle is an increased contractility soon followed by hypertrophy, with little or no increase in dimensions. This constitutes the beginning of this fascinating article that highlights the complex interactions between the heart and the normal or hypertensive pulmonary circulation, enhancing nonetheless the knowledge of the interplay to potentially offer guidance to differential diagnosis and management options. Robert Naeije, one of the best key leaders in the area of the complexities of the pulmonary vasculature, authorises this very thorough review of the pathophysiology of the mechanical abnormalities in the pulmonary vessels.

I invite you to take a look at these five timely topics. I very much hope you will enjoy these multifaceted aspects of current pulmonary medicine.

\section{REFERENCE LIST}

1. Vogelmeier CF, Criner GJ, Martinez FJ, et al. Global Strategy for the Diagnosis, Management, and Prevention of Chronic Obstructive Lung Disease 2017 Report. GOLD Executive Summary. Am J Respir Crit Care Med 2017;195:557-82. 